\title{
Potential for local resection with sentinel node basin dissection for early gastric cancer based on the distribution of primary sites
}

\author{
Junya Aoyama ${ }^{1} \cdot$ Hirofumi Kawakubo ${ }^{1}$ O Osamu Goto ${ }^{2} \cdot$ Tadaki Nakahara $^{3} \cdot$ Shuhei Mayanagi $^{1} \cdot$ Kazumasa Fukuda $^{1}$. \\ Koichi Suda ${ }^{1} \cdot$ Rieko Nakamura $^{1} \cdot$ Norihito Wada $^{1} \cdot$ Hiroya Takeuchi $^{1,4} \cdot$ Yuko Kitagawa ${ }^{1}$
}

Received: 11 June 2018 / Accepted: 5 August 2018 / Published online: 11 August 2018

(c) The International Gastric Cancer Association and The Japanese Gastric Cancer Association 2018

\begin{abstract}
Background Based on the sentinel node (SN) concept, function-preserving surgery with SN basin dissection (SNBD) can be performed for SN-negative early gastric cancers. Particularly, a resection area can be minimized when the SN basin and primary site are closely localized. The aim of this study was to compare probabilities of being candidates for local resection with SNBD based on tumor location among patients with early gastric cancer.

Methods We retrospectively analyzed 358 patients who underwent surgery with SN mapping for gastric cancer in our institution from November 1999 to April 2014. The proportion of patients who had a localized single basin and the distributions of the SN basins and primary sites were investigated. Patients with single basin drainage excluding remote sentinel node basin were considered as candidates for local resection with SNBD.

Results Of the 358 patients, 191 (53\%) patients were considered eligible for local resection with SNBD. Patients with tumors located in the upper third of the stomach were more likely candidates for local resection than those with tumors in other locations (upper third, 68\%; middle third, 50\%; and lower third, 51\%), whereas patients with tumors located in the anterior wall were less likely candidates than those with tumors other locations (anterior wall, 31\%; posterior wall, 58\%; greater curvature, $55 \%$; and lesser curvature, $57 \%$ ).

Conclusion We found that $>50 \%$ of the patients indicated for SN navigation surgery, particularly those with tumors in the upper third of the stomach, potentially could undergo partial resection with SNBD.
\end{abstract}

Keywords Minimally invasive surgery $\cdot$ Sentinel node navigation surgery $\cdot$ Local resection $\cdot$ Early gastric cancer

\section{Introduction}

The definition of early gastric cancer (EGC) is a tumor limited to the mucosa or submucosa with or without regional lymph node (LN) metastasis [1,2]. Currently, gastrectomy with D1 or D1 + lymphadenectomy is required to treat EGC with possible LN metastasis. However, the incidence of regional LN metastasis is 1-19\% only in EGC patients [3]. Therefore, for patients with node-negative EGC, standard lymphadenectomy may be too invasive. Lymphadenectomy with gastric resection could cause a decrease in the patients' quality of life (QOL) with post-gastrectomy symptoms, such as dumping syndrome [4] and body weight loss resulting from oral intake disturbance.

Faster improvement in postoperative gastric function is expected after function-preserving gastrectomy, such as partial gastrectomy, segmental gastrectomy, and proximal gastrectomy with reduced LN dissection [5-8]. We believe that 
the sentinel node ( $\mathrm{SN}$ ) concept is an attractive option for the provision of function-preserving surgery in EGC patients. In this concept, if there is no metastasis to SNs, which are identified by a radiolabeled colloid and/or a dye solution, the area of LN dissection can be minimized [9, 10]. This concept has already been validated by a prospective multicenter trial that included our institution [11], and the concept of SN basin dissection (SNBD) has also been proposed by several studies $[12,13]$. In general, the gastric LNs are divided into $5 \mathrm{SN}$ basins along with the main gastric arteries: left gastric artery (l-GA), right gastric artery (r-GA), right gastroepiploic artery (r-GEA), left gastroepiploic artery (l-GEA), and posterior gastric artery (p-GA) [12]. The 1-GA basin comprises the LN stations 1, 3a, and 7; the r-GA basin comprises the stations $3 \mathrm{~b}, 5$, and $8 \mathrm{a}$; and the r-GEA basin comprises the stations $4 \mathrm{~d}$ and 6 . The 1-GEA basin comprises the stations 4sa and 4sb, and the p-GA basin comprises the station 11p. Currently, SN navigation surgery (SNNS) with functionpreserving gastrectomy and SNBD is suggested to be safely applied to patients with cT1N0 gastric cancer with a primary lesion diameter of $\leq 4 \mathrm{~cm} \mathrm{[11].}$

In SNNS, local resection should be indicated for patients in which the distribution of cancers, including SN basins, is localized because the broad dissection of SN basins and minimal resection at the primary site may lead to the ischemic change of a relatively large remnant stomach. In addition, local resection should be applied to patients in which the primary site and SN basin are sufficiently close to each other to remove en-bloc because the separate resection of the primary lesion and SN basin may miss possibly cancer-positive connecting lymphatic ducts. Therefore, currently, local resection with SNBD is considered to be safe in patients having a single $\mathrm{SN}$ basin attached to the primary site [14].

However, the proportion of patients that are suitable for this procedure has not been investigated. In this study, we retrospectively analyzed patients who underwent surgery with SN mapping for EGC and determined the incidence and anatomical locations that are candidates for local resection with SNBD.

\section{Patients and methods}

\section{Enrollment of patients}

From November 1999 to April 2014, 545 patients who underwent surgery with SN mapping for gastric cancer at Keio University Hospital were enrolled. Patients with cT1N0 or cT2N0 gastric cancer were preoperatively indicated for surgery with SN mapping after evaluation using endoscopy and computed tomography. Clinical staging and pathological examination of tumors were performed according to the
Japanese Classification of Gastric Cancer [15] as proposed by the Japanese Gastric Cancer Association and the TNM classification [16]. Informed consent for SN mapping was obtained from all the patients before surgery, and this study was conducted after obtaining the approval of the Institutional Review Board of Keio University. We excluded patients with prior endoscopic treatment, multiple lesions, $\geq \mathrm{pT} 2,>4 \mathrm{~cm}$ in diameter, cancer-positive SNs, or without detection of SNs.

\section{Details of sentinel node navigation surgery}

We used a dual-tracer method with radiolabeled tin colloid and dye solution as described previously $[9,17]$. The day before surgery, $2.0 \mathrm{ml}$ of technetium- $99 \mathrm{~m}$ tin colloid solution (150 MBq) was injected into 4 quadrants of the submucosal layer surrounding the primary tumor lesion using an endoscopic puncture needle. The dye solution $(0.5 \%$ indocyanine green or $1 \%$ isosulfan blue) was injected via intraoperative endoscopy using the same method as the preoperative injection of the radioisotopes. We detected possible SNs that were stained blue $15 \mathrm{~min}$ after the injection of the dye solution. Simultaneously, radioactive LNs were detected by a hand-held gamma probe (GPS Navigator; RMD Instruments, Watertown, MA, USA). LNs with radioactivity $>10 \mathrm{x}$ background activity and/or blue-stained LNs were finally defined as the SNs. The SNs were examined pathologically during surgery. After SN mapping, all the patients underwent D2 or modified D2 gastrectomy according to the therapeutic guidelines recommended by The Japan Gastric Cancer Association [3].

\section{Statistical analysis}

SPSS statistics, version 24 (IBM Corporation, Armonk, NY, USA) was used to perform the statistical analyses. The differences in the categorical data between the 2 groups were evaluated by Pearson's chi square or Fisher's exact tests, and the continuous data were compared by performing the Student's $t$ test. We considered the differences to be statistically significant at $p<0.05$.

\section{Results}

The final study population comprised 358 patients. Of the 358 patients, 199 (56\%) had SNs limited to a single basin. Eight patients had basins on the contralateral side of the tumors. The basins were obviously separated from the primary tumors in these patients. Therefore, we excluded these patients from the candidates for partial resection with SNBD. Finally, 191 patients (53\%) with a single basin attached to the primary site were identified and were 
considered eligible for local resection with SNBD. The distribution of the basins were as follows: left gastric artery, $135(70.7 \%)$ patients; right gastric artery, $1(0.5 \%)$ patient; left gastroepiploic artery, 5 (2.6\%) patients; right gastroepiploic artery, 50 (26.2\%) patients; posterior gastric artery, 0 patient (0\%) (Fig. 1).

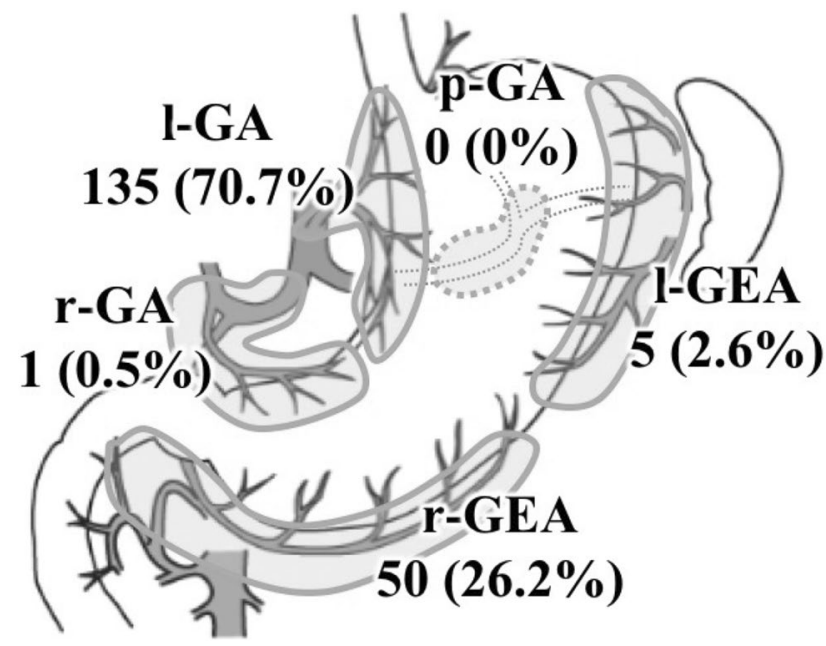

Fig. 1 Proportion of basins in patients with SNs limited to one basin attached to the primary tumor. The distribution of the basins were as follows: left gastric artery (l-GA), 135 (70.7\%) patients; right gastric artery (r-GA), $1(0.5 \%)$ patient; left gastroepiploic artery (l-GEA), $5(2.6 \%)$ patients; right gastroepiploic artery (r-GEA), $50(26.2 \%)$ patients; posterior gastric artery (p-GA), $0(0 \%)$ patients
Table 1 shows the patient characteristics. We defined the patients with a single basin drainage excluding remote $\mathrm{SN}$ basin as the potential group. There were significant differences in the lesion locations and circumferences of the primary lesion between the 2 groups. The proportion of lesions in the upper third of the stomach was higher in the potential group than in the non-potential group (24 vs. 13\%, $p=0.028)$. The percentage of lesions located on the anterior wall of the stomach was 9\% (17/191) in the potential group and 23\% (37/167) in the non-potential group, which was a significant difference $(p=0.006)$. There was no significant difference between the 2 groups in age, sex, pT factor, or tumor size.

Table 2 shows the probability of candidates for local resection with SNBD for each location of the primary lesion. Patients with tumors located in the upper third of the stomach were more likely candidates for local resection than those with tumors in other locations (upper third, $68 \%$; middle third, $50 \%$; and lower third, $51 \%$ ), whereas patients with tumors located in the anterior wall were less likely candidates than those with tumors in other locations (anterior wall, $31 \%$; posterior wall, $58 \%$; greater curvature, 55\%; and lesser curvature, 57\%). The probability was highest in patients with lesions located on the anterior wall of the upper third of the stomach. Among the tumors in the anterior wall, those located in the upper third of the stomach had a significantly higher probability than those located in the middle third ( 83 vs. $24 \%$, respectively; $p=0.017$ ) and those located in the lower third of the stomach (83 vs. $29 \%$,
Table 1 Clinicopathological characteristics

\begin{tabular}{|c|c|c|c|c|}
\hline & Total $(n=358)$ & $\begin{array}{l}\text { Potential for local resection } \\
\text { with SNBD }(n=191)\end{array}$ & $\begin{array}{l}\text { Non-potential } \\
(n=167)\end{array}$ & $p$ value \\
\hline Age (years, mean $\pm S D$ ) & $60.6 \pm 11.1$ & $61.6 \pm 10.7$ & $59.4 \pm 11.5$ & 0.060 \\
\hline Sex: $n(\%)$ & & & & 0.159 \\
\hline Male & $251(70 \%)$ & $140(73 \%)$ & $111(66 \%)$ & \\
\hline Female & $107(30 \%)$ & $51(27 \%)$ & $56(34 \%)$ & \\
\hline Lesion location: $n(\%)$ & & & & $0.028 *$ \\
\hline Upper third & $66(18 \%)$ & $45(24 \%)$ & $21(13 \%)$ & \\
\hline Middle third & $195(54 \%)$ & $97(51 \%)$ & $98(59 \%)$ & \\
\hline Lower third & $97(27 \%)$ & $49(26 \%)$ & $48(29 \%)$ & \\
\hline Circumference: $n(\%)$ & & & & $0.006^{*}$ \\
\hline Anterior wall & $54(15 \%)$ & $17(9 \%)$ & $37(23 \%)$ & \\
\hline Posterior wall & $101(28 \%)$ & $59(30 \%)$ & $42(26 \%)$ & \\
\hline Greater curvature & $56(16 \%)$ & $31(16 \%)$ & $25(15 \%)$ & \\
\hline Lesser curvature & $147(41 \%)$ & $84(44 \%)$ & $63(38 \%)$ & \\
\hline pT factor: $n(\%)$ & & & & 0.914 \\
\hline pT1a & $223(62 \%)$ & $125(63 \%)$ & $98(62 \%)$ & \\
\hline pT1b & $135(38 \%)$ & $74(37 \%)$ & $61(38 \%)$ & \\
\hline Tumor size (median $\pm \mathrm{SD})$ & $26.2 \pm 11.5$ & $26.7 \pm 11.6$ & $25.7 \pm 11.4$ & 0.380 \\
\hline
\end{tabular}


Table 2 Proportion of candidates for local resection with SNBD for each location of the primary lesion (number of eligible patients for local resection with SNBD or number of SNNS indicated patients, \%)

\begin{tabular}{llllll}
\hline & Ant & Post & Gre & Less & Total \\
\hline $\mathrm{U}$ & $5 / 6$ & $18 / 26$ & $0 / 1$ & $22 / 33$ & $45 / 66$ \\
& $83 \%$ & $69 \%$ & $0 \%$ & $67 \%$ & $68 \%$ \\
$\mathrm{M}$ & $8 / 34$ & $26 / 52$ & $14 / 31$ & $49 / 78$ & $97 / 195$ \\
& $24 \%$ & $50 \%$ & $45 \%$ & $63 \%$ & $50 \%$ \\
$\mathrm{~L}$ & $4 / 14$ & $15 / 23$ & $17 / 24$ & $13 / 36$ & $49 / 97$ \\
& $29 \%$ & $65 \%$ & $71 \%$ & $36 \%$ & $51 \%$ \\
\multirow{2}{*}{ Total } & $17 / 54$ & $59 / 101$ & $31 / 56$ & $84 / 147$ & $191 / 358$ \\
& $31 \%$ & $58 \%$ & $55 \%$ & $57 \%$ & $53 \%$ \\
\hline
\end{tabular}

Italicized parts exceeded the average value $(191 / 358,53 \%)$

$S N B D$ sentinel node basin dissection, $S N N S$, sentinel node navigation surgery, $U$ upper third of the stomach, $M$ middle third of the stomach, $L$ lower third of the stomach, Ant anterior side of the stomach, Post posterior side of the stomach, Gre greater curvature side of the stomach, Less lesser curvature side of the stomach

respectively; $p=0.042$ ). Other locations in the upper third of the stomach also had high probabilities, except those in the greater curvature side. The probabilities for lesions located in the upper third of the stomach were as follows: anterior wall, $83 \%$; posterior wall, 69\%; greater curvature, $0 \%$; and lesser curvature, $67 \%$. The patients with tumors in the greater curvature of the lower third had a higher probability $(71 \%)$ as well.

\section{Discussion}

The purpose of this study was to clarify potential EGCs for local resection with SNBD according to lesion location. We demonstrated that $53 \%$ of the SNNS patients were potential candidates for local resection with SNBD. Patients with tumors located at the upper third of the stomach were more likely candidates than those with tumors in other location (upper third, 68\%; middle third, 50\%; and lower third, 51\%).

To the best of our knowledge, this is the first report to investigate factors predictive of potentially beneficial treatment for local resection in SNNS. There are various options of primary tumor resection in SNNS [8]. Among them, full-thickness partial resection can minimize the resection area according to lesion size and is an ideal type of limited surgery [18-20]. However, it is assumed that wide resection cannot be avoided depending on the distribution of basins because of concern about necrosis of the remnant stomach. Nevertheless, our results suggested that local resection could be beneficial for more than half of patients in SNNS, which provides sufficient support for function-preserving surgery based on the SN concept.
Our results showed that the 1-GA basin was present in $70.7 \%$ of the 191 patients with SNs limited to single basin attached to the primary tumor. As reported by Tokunaga et al. [21], the major lymphatic drainage routes of the stomach were associated with 1-GA and r-GEA. In addition, they described the lymphatic regions covered by $1-G A$ and $r-G E A$ overlapped in the middle and lower third of the stomach [21]. The lymphatic regions covered by $1-G A$ or r-GEA and not overlapping each other, which almost coincided with the location considered to be a good indicator in the present study, e.g., the upper body except the greater curvature was covered by only 1-GA and the greater curvature in the lower body was covered by only r-GEA. On the other hand, patients with tumors at regions covered by both 1-GA and r-GEA, such as the tumors located at the anterior wall in the middle and lower bodies and the lesser curvature in the lower body could not be considered good candidates. The tumors located at the greater curvature in the middle and lower third of the stomach has been considered suitable for partial resection with SNBD because it was relatively easy to perform partial resection and basin dissection without nerve injury $[8,9]$. However, our results showed that $>50 \%$ of the patients with tumors located at the greater curvature in the middle body failed to become candidates, with one reason being that SNs of tumors at the greater curvature in the middle body tended to be detected not only in the r-GEA basin but also in the 1-GEA basin. The 1-GEA basin was detected in $39 \%(12 / 31)$ of these cases, whereas the patients with tumors located at the greater curvature of lower body did not have SNs in the 1-GEA basin at all. Furthermore, the 1-GA or r-GA basins were identified in 32\% (10/31) of the cases with tumors located at the greater curvature in the middle body.

Laparoscopic endoscopic cooperative surgery (LECS) is an ideal full-thickness partial resection technique [22, 23]. This technique makes it possible to minimize the extent of gastric resection by visualizing a demarcation line of the lesion from the inside of the lumen endoscopically. With the aim of avoiding the risk of iatrogenic dissemination, several approaches have been proposed, such as inverted LECS [24], non-exposed endoscopic wall-inversion surgery [14, 18-20, 25], and a combined laparoscopic and endoscopic approach for neoplasia with a non-exposure technique [26]. Comparison between standard gastrectomy with lymphadenectomy and function-preserving surgery with SNBD has been recently reported $[27,28]$, although these studies did not include cases with these procedures. However, the feasibility of these procedures for gastric submucosal tumors and gastric cancer has been demonstrated previously [8, 19, 24, 29]. SNNS-based function-preserving and limited surgery is expected to be more effective by using local resection techniques, such as these methods; however, there may be some technical limitations. Clinical validation of efficacy and safety of these procedures requires further investigation. 
In our study, the basins containing SNs were obviously separated from the primary tumor in 8 patients. Currently, we consider that local resection should not be performed in these patients because the primary lymph vessels that may contain cancer cells remain intact. Although there has been no evidence of recurrence from the remnant lymph duct and the possibility that cancer cells remain in the lymph vessels is extremely low in $\mathrm{SN}$-negative patients, a previous report speculated that an ignorable amount of cancer cells may remain $[30,31]$. Therefore, to obtain theoretically complete resection, the identified lymph vessels should be resected with the sero-muscular layer [14]. Several studies have reported how to visualize not only SNs but also lymph vessels during surgery by using indocyanine green fluorescence imaging [32-35]. Therefore, the resection of lymph vessels with the sero-muscular layer would be possible technically, although the oncological significance remains unknown. To establish local resection with SNBD as an optimal procedure, future long-term observations are necessary.

There were several limitations in this study. First, this was a retrospective study in patients collected according to postoperatively diagnosed pathological findings. Second, local resection with SNBD may be technically difficult in some cases even though it is possible theoretically. Third, all lesions attached to a single basin should not always be partially resected because of possible deformity or stasis following surgery [8]. Further prospective investigation of long-term outcomes is required to determine the safety and efficacy, particularly for patient's QOL and risk of recurrence, of local resection with SNBD.

In conclusion, we found that more than half of the SNNS patients, particularly those with tumors in the upper third of the stomach, were potential candidates for partial resection with SNBD. For patients with tumors in the upper third of the stomach, avoiding total gastrectomy or proximal gastrectomy while preserving the cardiac function by local resection with SNBD is advantageous. Furthermore, the extent of residual stomach deformation after partial resection in the upper region is less. This study would not be very useful in the decision-making during surgery because actual surgery depends upon the intraoperative $\mathrm{SN}$ status even if surgeons preoperatively know this information. However, the present study showed that the location of tumors could be a useful predictive factor for performing function-preserving surgery with SN navigation.

\section{Compliance with ethical standards}

Ethical approval All the procedures were followed in accordance with the ethical standards of the responsible committee on human experimentation (institutional and national) and with the Helsinki Declaration of 1964 and later versions.
Informed consent Informed consent was obtained from all patients for inclusion in the study.

Conflict of interest Yuko Kitagawa received designated donation from Daiichi Sankyo Co., Ltd.

\section{References}

1. Carter KJ, Schaffer HA, Ritchie WPJ. Early gastric cancer. Ann Surg. 1984;199:604-9.

2. Everett SM, Axon AT. Early gastric cancer in Europe. Gut. 1997:41:142-50.

3. Japanese Gastric Cancer A. Japanese gastric cancer treatment guidelines 2014 (ver. 4). Gastric Cancer. 2017;20:1-19.

4. Mine S, Sano T, Tsutsumi K, Murakami Y, Ehara K, Saka M, et al. Large-scale investigation into dumping syndrome after gastrectomy for gastric cancer. J Am Coll Surg. 2010;211:628-36.

5. Hiki N, Nunobe S, Kubota T, Jiang X. Function-preserving gastrectomy for early gastric cancer. Ann Surg Oncol. 2013;20:2683-92.

6. Nunobe S, Hiki N. Function-preserving surgery for gastric cancer: current status and future perspectives. Transl Gastroenterol Hepatol. 2017;2:77.

7. Saito T, Kurokawa Y, Takiguchi S, Mori M, Doki Y. Current status of function-preserving surgery for gastric cancer. World J Gastroenterol. 2014;20:17297-304.

8. Takeuchi H, Goto O, Yahagi N, Kitagawa Y. Function-preserving gastrectomy based on the sentinel node concept in early gastric cancer. Gastric Cancer. 2017;20:53-9.

9. Takeuchi H, Kitagawa Y. New sentinel node mapping technologies for early gastric cancer. Ann Surg Oncol. 2013;20:522-32.

10. Takeuchi H, Kitagawa Y. Sentinel node navigation surgery in patients with early gastric cancer. Dig Surg. 2013;30:104-11.

11. Kitagawa Y, Takeuchi H, Takagi Y, Natsugoe S, Terashima M, Murakami N, et al. Sentinel node mapping for gastric cancer: a prospective multicenter trial in Japan. J Clin Oncol. 2013;31:3704-10.

12. Kinami S, Fujimura T, Ojima E, Fushida S, Ojima T, Funaki H, et al. PTD classification: proposal for a new classification of gastric cancer location based on physiological lymphatic flow. Int $\mathrm{J}$ Clin Oncol. 2008;13:320-9.

13. Miwa K, Kinami S, Taniguchi K, Fushida S, Fujimura T, Nonomura A. Mapping sentinel nodes in patients with early-stage gastric carcinoma. Br J Surg. 2003;90:178-82.

14. Goto O, Takeuchi H, Kawakubo H, Matsuda S, Kato F, Sasaki M, et al. Feasibility of non-exposed endoscopic wall-inversion surgery with sentinel node basin dissection as a new surgical method for early gastric cancer: a porcine survival study. Gastric Cancer. 2015;18:440-5.

15. Association JGC. Japanese classification of gastric carcinoma: 3rd English edition. Gastric Cancer. 2011;14:101-12.

16. UCC. TNM classification of malignant tumours. Weinheim: Wiley; 2009.

17. Kitagawa Y, Fujii H, Mukai M, Kubota T, Ando N, Watanabe $\mathrm{M}$, et al. The role of the sentinel lymph node in gastrointestinal cancer. Surg Clin North Am. 2000;80:1799-809.

18. Goto O, Mitsui T, Fujishiro M, Wada I, Shimizu N, Seto Y, et al. New method of endoscopic full-thickness resection: a pilot study of non-exposed endoscopic wall-inversion surgery in an ex vivo porcine model. Gastric Cancer. 2011;14:183-7.

19. Goto O, Takeuchi H, Kawakubo H, Sasaki M, Matsuda T, Matsuda S, et al. First case of non-exposed endoscopic wall-inversion 
surgery with sentinel node basin dissection for early gastric cancer. Gastric Cancer. 2015;18:434-9.

20. Mitsui T, Niimi K, Yamashita H, Goto O, Aikou S, Hatao F, et al. Non-exposed endoscopic wall-inversion surgery as a novel partial gastrectomy technique. Gastric Cancer. 2014;17:594-9.

21. Tokunaga M, Ohyama S, Hiki N, Fukunaga T, Yamada K, Sano $\mathrm{T}$, et al. Investigation of the lymphatic stream of the stomach in gastric cancer with solitary lymph node metastasis. World J Surg. 2009;33:1235-9.

22. Hiki N, Nunobe S, Matsuda T, Hirasawa T, Yamamoto Y, Yamaguchi T. Laparoscopic endoscopic cooperative surgery. Dig Endosc. 2015;27:197-204.

23. Hiki N, Yamamoto Y, Fukunaga T, Yamaguchi T, Nunobe S, Tokunaga M, et al. Laparoscopic and endoscopic cooperative surgery for gastrointestinal stromal tumor dissection. Surg Endosc. 2008;22:1729-35.

24. Nunobe S, Hiki N, Gotoda T, Murao T, Haruma K, Matsumoto $\mathrm{H}$, et al. Successful application of laparoscopic and endoscopic cooperative surgery (LECS) for a lateral-spreading mucosal gastric cancer. Gastric Cancer. 2012;15:338-42.

25. Mitsui T, Goto O, Shimizu N, Hatao F, Wada I, Niimi K, et al. Novel technique for full-thickness resection of gastric malignancy: feasibility of nonexposed endoscopic wall-inversion surgery (NEWS) in porcine models. Surg Laparosc Endosc Percutan Tech. 2013;23:e217-21.

26. Inoue H, Ikeda H, Hosoya T, Yoshida A, Onimaru M, Suzuki M, et al. Endoscopic mucosal resection, endoscopic submucosal dissection, and beyond: full-layer resection for gastric cancer with nonexposure technique (CLEAN-NET). Surg Oncol Clin N Am. 2012;21:129-40.

27. Park JY, Kim YW, Ryu KW, Nam BH, Lee YJ, Jeong SH, et al. Assessment of laparoscopic stomach preserving surgery with sentinel basin dissection versus standard gastrectomy with lymphadenectomy in early gastric cancer-a multicenter randomized phase III clinical trial (SENORITA trial) protocol. BMC Cancer. 2016;16:340.
28. Arigami T, Uenosono Y, Yanagita S, Okubo K, Kijima T, Matsushita $\mathrm{D}$, et al. Clinical application and outcomes of sentinel node navigation surgery in patients with early gastric cancer. Oncotarget. 2017;8:75607-16.

29. Kato M, Uraoka T, Isobe Y, Abe K, Hirata T, Takada Y, et al. A case of gastric adenocarcinoma of fundic gland type resected by combination of laparoscopic and endoscopic approaches to neoplasia with non-exposure technique (CLEAN-NET). Clin J Gastroenterol. 2015;8:393-9.

30. Shimizu Y, Takeuchi H, Sakakura Y, Saikawa Y, Nakahara T, Mukai M, et al. Molecular detection of sentinel node micrometastases in patients with clinical N0 gastric carcinoma with real-time multiplex reverse transcription-polymerase chain reaction assay. Ann Surg Oncol. 2012;19:469-77.

31. Takeuchi H, Ueda M, Oyama T, Shimizu Y, Kitagawa Y. Molecular diagnosis and translymphatic chemotherapy targeting sentinel lymph nodes of patients with early gastrointestinal cancers. Digestion. 2010;82:187-91.

32. Miyashiro I, Miyoshi N, Hiratsuka M, Kishi K, Yamada T, Ohue $\mathrm{M}$, et al. Detection of sentinel node in gastric cancer surgery by indocyanine green fluorescence imaging: comparison with infrared imaging. Ann Surg Oncol. 2008;15:1640-3.

33. Schaafsma BE, Mieog JS, Hutteman M, van der Vorst JR, Kuppen $\mathrm{PJ}$, Lowik CW, et al. The clinical use of indocyanine green as a near-infrared fluorescent contrast agent for image-guided oncologic surgery. J Surg Oncol. 2011;104:323-32.

34. Tajima Y, Murakami M, Yamazaki K, Masuda Y, Kato M, Sato A, et al. Sentinel node mapping guided by indocyanine green fluorescence imaging during laparoscopic surgery in gastric cancer. Ann Surg Oncol. 2010;17:1787-93.

35. Tajima Y, Yamazaki K, Masuda Y, Kato M, Yasuda D, Aoki T, et al. Sentinel node mapping guided by indocyanine green fluorescence imaging in gastric cancer. Ann Surg. 2009;249:58-62. 